\title{
Prevalence of enamel defects and associated risk factors in both dentitions in preterm and full term born children
}

Vanessa Resende Nogueira CRUVINEL', Danuze Batista Lamas GRAVINA', Tatiana Degani Paes Leme AZEVEDO', Catharina Siqueira de REZENDE², Ana Cristina Barreto BEZERRA ${ }^{3}$, Orlando Ayrton de TOLEDO 4

1- DDS, MSc, Professor, Department of Pediatric Dentistry, School of Dentistry, Catholic University of Brasília, Brasília, DF, Brazil.

2- Private Practice, Catholic University of Brasília, Brasília, DF, Brazil.

3- DDS, MSc, PhD, Associate Professor, Department of Pediatric Dentistry, School of Dentistry, University of Brasília, Brasília, DF, Brazil.

4- DDS, PhD, Associate Professor, Department of Pediatric Dentistry, School of Dentistry, University of Brasília, Brasília, DF, Brazil.

Corresponding address: Vanessa Resende Nogueira Cruvinel - Universidade Católica de Brasília - Curso de Odontologia Colônia Agrícola Samambaia, Rua 01 - Chácara 99 - Casa C - 72110600 - Taguatinga - DF - Brasil - Fone: 556135615376

- Fax: 5561 3352-4130 - e-mail: vanessarcruvinel@gmail.com

Received: September 09, 2010 - Modification: August 01, 2011 - Accepted: August 25, 2011

\section{ABSTRACT}

$\mathrm{O}$ bjectives: The aim of this study was to evaluate the prevalence of enamel defects and their risk factors on primary and permanent dentitions of prematurely born children and full-term born children born at Regional Hospital of Asa Sul, Brasília, DF, Brazil. Material and Methods: Eighty 5-10-year-old children of both genders were examined, being 40 born prematurely (G1) and 40 born full term (G2). The demographic variables, medical history and oral health behaviors were retrieved using a questionnaire and data obtained from clinical examination were recorded. The teeth were examined and the presence of enamel defects was diagnosed according to the DDE Index and registered in odontograms. Subsequently, the defects were categorized in four groups according to one of the criteria proposed in 1992 by the FDI Commission on Oral Health, Research and Epidemiology. Kruskal-Wallis, Chi-square, Kappa, Mann-Whitney tests and logistic regression were used for statistical analysis. Results: $75 \%$ of total sample had enamel defects. There was a major prevalence of hypoplasia of the enamel in $G 1(p<0.001)$. There was a significant relationship between low weight and presence of the imperfections on the enamel in G1 on the primary dentition. The logistic regression model showed that the other risk factors such as monthly per capita family income, educational level, dietary and hygiene habits, fluoride exposure, trauma, and diseases were not associated with enamel defects and caries. Conclusions: Pre-term labor can be a predisposing factor for the presence of the enamel hypoplasia in the primary dentition.

Key words: Dental enamel. Dental enamel hypoplasia. Premature infant. Deciduous tooth. 


\section{INTRODUCTION}

According to the World Health Organization $(\mathrm{WHO})^{21}$, a newborn of less than 37 weeks gestation or born within fewer than 259 days after the last menstrual period is considered premature or preterm. Prematurity can be classified as mild, when the baby is born between the $35^{\text {th }}$ and $36^{\text {th }}$ weeks of gestation; moderate, if the birth occurs between the $31^{\text {st }}$ and $34^{\text {th }}$ weeks; or extreme, if the gestational age is less than or equal to 30 weeks.

The birth of pre-term and/or low birth weight newborns represents a public health problem with increased economic, social, family, and individual costs $^{10}$. Preventive and health promoting measures become necessary in order to improve the quality of life of these children. As such, knowing the risk factors to which they are subject is of fundamental importance for the adoption of such measures.

Low gestational age and low birth weight are the main factors that determine the incidence of neonatal complications. Among the most frequent complications are neonatal rickets, hypocalcemia, perinatal anoxia, anemia, infections, and metabolic, renal, respiratory, cardiovascular and hematological diseases. In these circumstances the use of various drug therapies and, frequently, orotracheal or laryngoscopic intubation to overcome respiratory difficulties, are necessary. These pathologies, whether or not associated with ventilatory support, may cause anomalies in the oral structures of these babies. In a previous study, low birth weight preterm infants presented a higher prevalence of hypoplasia than normal birth weight controls, and the most affected primary teeth by hypoplasia were maxillary incisors ${ }^{7}$.

Among the most prevalent oral alterations in these children are hypoplasias and opacities of the dental enamel ${ }^{6,8}$. The formation of these defects is related to disorders during amelogenesis ${ }^{6}$. The term during which the attack on the ameloblasts occurs is very important to the location and the appearance of enamel defects. The final enamel shows a record of injuries received during the development of the teeth, which may appear as hypoplasia of the enamel, diffuse or marked opacity, and hypomineralization of the ename ${ }^{20}$. Enamel hypoplasia is the most common of the changes in human tooth development, with relevant clinical implications due to esthetic reasons, symptoms involved, susceptibility to caries, and also to the difficulty of treatment in many cases. This condition occurs as a direct result of disorders of metabolism of the ameloblast layers of the enamel organ ${ }^{19}$.

Hypoplastic areas are reported as being highly susceptible to dental caries co $^{3,9,15}$ because, through ultrastructural analysis, they have shown enamel with less mineralization, more porosity, with irregular surfaces allowing the accumulation of bacteria which is favorable to the development of colonies of Streptococcus mutans, resulting in carious lesions $\mathbf{3}^{3,11,13}$.

Knowing that children born prematurely present alterations in the dental structures, and that these can be related to the development of carious lesions, we must focus our attention on certain risk groups within which they fall. Given this evidence, this study aimed at evaluating the prevalence of enamel defects in the dentition of children born prematurely compared with those born full-term, and the main risk factors associated with those defects.

\section{MATERIAL AND METHODS}

\section{Sample}

This was a cross-sectional study involving children born in the Regional Hospital of Asa Sul HRAS-DF, a public hospital from the Brazilian Unified National Health System (SUS), considered to be a reference for high-risk births.

The sample for this study comprised 80 children of both genders, born in this hospital between 2000 and 2004, between 5 and 10 years of age, with birth weights that ranged from $605 \mathrm{~g}$ to 4300 g. The children were taken from a case-control study done in 2004, aimed at comparing the oral alterations present in 96 premature babies and 96 full-term babies born in this maternity hospital, making a total of 192 children. In calculating the initial sample ( $1^{\text {st }}$ study), a confidence interval of $95 \%$, with a margin of error of $10 \%$ (more or less) ${ }^{8}$, was considered. Of the children analyzed in the previous study, 98 were selected by having their charts updated with address and telephone number. Of these, 18 chose not to participate in the research. To calculate this sample, we took into account a 10 to $15 \%$ annual loss, which can be expected in longitudinal epidemiological studies. This study was completed with 80 children.

Children born before the $37^{\text {th }}$ gestational week were classified as premature, according to the WHO criteria ${ }^{21}$. In this sample, 40 children were premature and 40 children were full-term. The children were categorized according to birth weight as: very low weight (less than 30 weeks, $1500 \mathrm{~g}$ ); low weight (between 1500 and $2500 \mathrm{~g}$, between $31^{\text {st }}$ and $34^{\text {th }}$ weeks); and normal weight (above $2500 \mathrm{~g}$ and $>38$ weeks $)^{17}$.

Information about the birth conditions, such as number of weeks and birth weight as well as medical history of the mother and child, were taken from the registration forms of the first study, whose data were obtained from the hospital medical charts.

Prior to the beginning of this study, the mothers received all the explanations concerning the 
monitoring and dental evaluations of the children, and those who wished to participate signed an informed consent form and filled out an individual questionnaire, which was tested against the pilot study.

\section{Use of the questionnaire}

The questionnaire was used as an interview technique, incorporating both open and closed questions, by a previously trained interviewer. This technique was chosen as it had been shown to be an accurate research instrument for the investigation of health habits and conditions. The results gained here have been shown, in these cases, to be more reliable that those obtained through fill-in forms ${ }^{16}$.

The questionnaire contained three parts:

\section{General information}

This included name, age, gender, date of birth, family income, place of residence, and parents' occupations.

\section{Medical history}

Information regarding probable risk factors for opacity and hypoplasia were collected, such as: systemic infectious diseases and rashes which occurred in the first 3 years of life, such as pneumonia, tonsillitis, ear infections, chickenpox, rubella, measles; history of injury and low birth weight.

In relation to the categorization of family socioeconomic status, the standard variables as defined by the National Research of Domestic Samples (PNAD), which is done annually and portrays the socioeconomic situation of the Brazilian population, were considered. The variables defined by the PNAD include: family (group of persons connected by parentage, domestic dependency or norms of familiarity, who reside in the same household); literate persons (those who are capable of reading and writing at least a simple message in the familiar language); level or number of years of formal education (school grade and level - elementary, secondary, or college - attended, considering the last grade completed successfully); monthly per capita family income (monthly family income divided by the number of people in the family). For the determination of income, the value of the Brazilian national minimum wage in force during the months referenced in this study was considered - R \$465.00. Starting with this last indicator, families were initially classified into three groups: below poverty level (per capita household income less than $1 / 4$ of the minimum wage), at poverty level (per capita household income between $1 / 4$ and $1 / 2$ minimum wage), and above $1 / 2$ per capita minimum wage. Subsequently, these variables were categorized in 2 groups: $1 / 2$ minimum wage and above $1 / 2$ minimum wage, because only 6 and 5 children of $\mathrm{G} 1$ and $\mathrm{G} 2$, respectively, showed income less than $1 / 4$ minimum wage.

\section{Clinical exam}

This procedure was done at the Dental Clinic of the Catholic University of Brasilia, under artificial light, using dental mirror number 3 and properly sterilized gauze for cleaning. The patient was seated in the dental chair and submitted to prophylaxis with a Robson electric toothbrush and prophylactic paste manufactured by Vigodent. Afterward, the dental surfaces were dried with streams of air.

The dental surfaces were examined to verify the presence of structural defects in the enamel. The tooth was considered present when any part of the clinical crown was exposed in the oral cavity. When there was doubt about the presence of defect, the tooth was considered normal. Defects measuring less than one millimeter in diameter were excluded ${ }^{9,12,14}$.

All evaluations were performed by the same examiner, who was trained in the pilot study. To establish the degree of intra-examiner agreement, the Kappa index of $10 \%$ of the sample was used, as suggested by the $\mathrm{WHO}^{21}$. Re-examination was done 1 week after the initial examination (Kappa $=0.90$ for opacities, hypoplasias and white spots).

Codes for the diagnosis of enamel defects were used, according to the criteria of the Index of Developmental Defects of Dental Enamel (DDE Index $)^{6}$.

Teeth were examined by quadrants for hypoplasia and opacities of the enamel in the following order: right maxillary, left maxillary, right mandibular, and left mandibular. The number of teeth with defects was registered, as well as the total number of primary and erupted permanent teeth.

Hypoplasia of the enamel was defined as a break in the continuity of the enamel with a reduction in the layers, creating grooves or depressions. Opacity was defined as a change in the translucence of the enamel, or a qualitative defect that could vary in color from white to yellow and brown. The extent, type and color of the defect were registered. When hypoplasia and opacity were found in the same tooth, the defect was registered as hypoplasia ${ }^{8}$.

These data were presented in relation to the number of children that showed defects in the enamel, and in relation to the number of affected teeth per child. Subsequently, the defects were categorized in four groups: 1- marked opacity (codes 1 and 2), 2- diffuse opacity (codes 3 to 6), 3- hypoplasia (codes 7 and 8), and 4- other defects (code 9) according to one of the criteria proposed in 1992 by the FDI Commission on Oral Health, Research and Epidemiology. 


\section{Analysis of data}

The Mann-Whitney non-parametric test was used to compare the results of the scores of opacity and hypoplasia. A significance level of $5 \%$ was used for analytical purposes.

An adjustment was made to the multivariate logistical regression, in order to examine possible risk factors associated with occurrence of opacity and hypoplasia. Occurrence of opacity (Yes $=1$, $\mathrm{No}=0$ ) and occurrence of hypoplasia (Yes $=1, \mathrm{No}=0$ ) were used as the dependent variables. Type of birth (Premature $=1$, Full-term $=0$ ) was considered as a preponderant risk factor, and the following variables were considered as potential confounding factors: mother's education level ( $<8$ years of $s c h o o l=1$, 8 years of school $=0)$, monthly per capita family income ( $<0.5$ minimum wage $=1,0.5$ minimum wage $=0$ ), dental trauma (had trauma $=1$, did not have trauma $=0$ ), infectious diseases ( $Y e s=1$, $\mathrm{No}=0)$, and rashes (Yes=1, No=0).

Initially, a bivariate analysis was carried out between the dependent variable and all the independent, confounding variables with the aim of identifying those which could be related to the dependent variable. In this phase, significance was considered when the $p$-value was less than 0.25 . Subsequently, a multivariate analysis was carried out only with the variables selected in the bivariate process, plus the preponderant independent variable as risk factor. In this phase a significance level of $5 \%$ was used.
This study was approved by the Ethics Committee of the Catholic University of Brasilia (UCB) under number $161 / 2008$.

\section{RESULTS}

The ages of the children ranged from 5 to 10 years, with a mean of 6.3 years for the premature group and 7.6 years for the full-term group, with a significant different between the groups $(p=0.0003)$. The mean weight for premature children was $1255 \mathrm{~g}$, and for full-term children was $3398 \mathrm{~g}$.

Considering the 80 children examined, approximately $72.5 \%$ of the sample presented at least one tooth with enamel defect. According to the number of teeth affected, the groups presented the following mean scores in relation to the 2 types of defects: Presence of Opacity, G1 - 2.3 and G2 - 2.8 teeth $(p=0.8624)$; and Hypoplasia, G1 - 1.1 and $G 2-0.1$ teeth with this defect $(p=0.0011)$.

No significant difference was found in the prevalence of enamel defects when children from G1 and G2 were divided by gender, with values of $p=0.5712$ for opacity and $p=1.000$ for hypoplasia.

The percentage of children in $\mathrm{G} 1$ and $\mathrm{G} 2$ who presented opacity and hypoplasia was presented at Table 1 . The percentage of primary and permanent teeth with opacity and hypoplasia in $\mathrm{G} 1$ and $\mathrm{G} 2$ was detailed at Table 2 and 3, respectively.

There was a statistically significant difference between the groups for marked opacity $(p=0.0174)$

Table 1- Percentage of children in G1 and G2 who presented opacity and hypoplasia

\begin{tabular}{|c|c|c|c|c|c|c|c|c|}
\hline & & \multicolumn{2}{|c|}{ Premature- G1 } & \multicolumn{2}{|c|}{ Full-term- G2 } & \multicolumn{2}{|c|}{ Total } & \multirow[t]{2}{*}{$p$} \\
\hline & & $n$ & $\%$ & $n$ & $\%$ & $n$ & $\%$ & \\
\hline \multirow[t]{2}{*}{ Opacity } & Yes & 26 & 65.0 & 25 & 62.5 & 51 & 63.75 & 0.8161 \\
\hline & No & 14 & 35.0 & 15 & 37.5 & 29 & 36.25 & \\
\hline \multirow[t]{2}{*}{ Hypoplasia } & Yes & 15 & 37.5 & 3 & 7.5 & 18 & 22.50 & 0.0013 \\
\hline & No & 25 & 62.5 & 37 & 92.5 & 62 & 77.50 & \\
\hline
\end{tabular}

Table 2- Percentage of primary teeth with opacity and hypoplasia in G1 and G2

\begin{tabular}{|c|c|c|c|c|c|}
\hline \multirow[t]{2}{*}{ Sample } & \multirow[t]{2}{*}{ Groups of teeth } & \multicolumn{2}{|c|}{ Opacity } & \multicolumn{2}{|c|}{ Hypoplasia } \\
\hline & & $\mathbf{n}$ & $\%$ & $\mathbf{n}$ & $\%$ \\
\hline \multirow[t]{3}{*}{ Total } & Incisor & 2 & 0.7 & 23 & 8 \\
\hline & Canine & 17 & 6.1 & 10 & 3.6 \\
\hline & Molar & 43 & 7.5 & 13 & 2.3 \\
\hline \multirow[t]{3}{*}{ G1 (premature) } & Incisor & 2 & 1 & 23 & 11.8 \\
\hline & Canine & 12 & 8.4 & 9 & 6.3 \\
\hline & Molar & 29 & 9.8 & 10 & 3.4 \\
\hline \multirow[t]{3}{*}{ G2 (full-term) } & Incisor & 0 & 0 & 0 & 0 \\
\hline & Canine & 5 & 5.5 & 1 & 0.75 \\
\hline & Molar & 14 & 5 & 3 & 1.06 \\
\hline
\end{tabular}


and hypoplasia $(\mathrm{p}<0.001)$, for primary teeth (Figure 1). However, there was no significant difference for any type of defect for permanent teeth (Figure 2).

Controlling for the effects of the variables that could be considered a probable risk factor for the occurrence of defects in the enamel such as: mother's level of education (0.5777), per capita income $(0.2442)$, trauma $(p=0.9784)$, infectious diseases $(p=0.4906)$, and rashes $(p=0.9571)$, the type of birth was not a risk factor for the occurrence of opacity ( $p=0.8161)$ with odds ratio equaling 1.11 ( 0.45 to 2.77 ). However, the type of birth was a risk factor for the occurrence of hypoplasia $(p=0.0034)$ with odds ratio equaling 7.4 (1.94 to 28.25 ).

Table 3- Percentage of permanent teeth with opacity and hypoplasia in G1 and G2

\begin{tabular}{cccccc}
\hline Sample & Groups of teeth & Opacity & \multicolumn{3}{c}{ Hypoplasia } \\
& & $\mathbf{n}$ & $\mathbf{n}$ & $\mathbf{n}$ & $\%$ \\
\hline \multirow{2}{*}{ Total } & Incisor & 72 & 20 & 3 & 0.5 \\
& Canine & 3 & 13.6 & 0 & 0 \\
\hline \multirow{2}{*}{ G1 (premature) } & Molar & 54 & 25 & 1 & 0.1 \\
& Incisor & 16 & 13 & 2 & 0.8 \\
& Canine & 0 & 0 & 0 & 0 \\
\hline \multirow{2}{*}{ G2 (full-term) } & Molar & 27 & 32.5 & 1 & 1.2 \\
& Incisor & 56 & 24 & 2 & 0.5 \\
& Canine & 3 & 18.7 & 0 & 0 \\
\hline & Molar & 27 & 20.4 & 0 & 0 \\
\hline
\end{tabular}

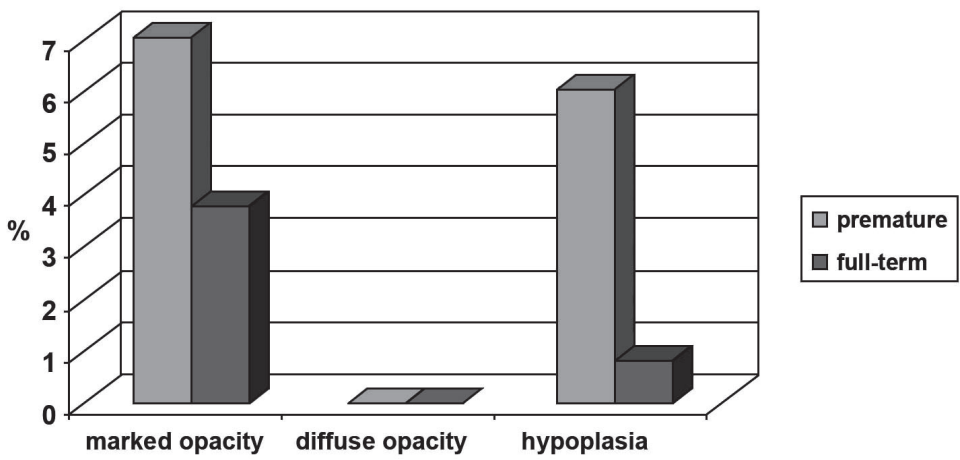

Figure 1- Percentage of marked opacity, diffuse opacity and hypoplasia in primary teeth

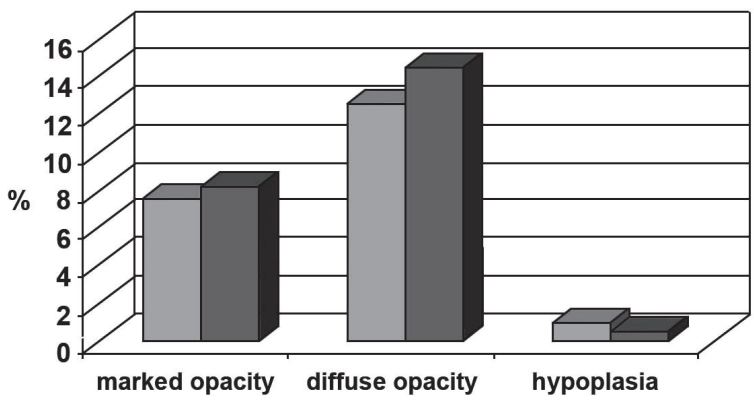

$\square$ premature

Figure 2 - Percentage of marked opacity, diffuse opacity and hypoplasia in permanent teeth 


\section{DISCUSSION}

In Brazil, developmental defects of enamel are not studied enough although they result in esthetic problems, dental sensibility, and are predisposing factors for dental caries. Prematurity has been described as one of the causes for the appearance of enamel defects ${ }^{6,8}$. The etiological factors for these problems are multiple, and they range from the conception of the baby through the first years of life, making it difficult to demonstrate the association between the variables. The scarcity of studies that evaluate permanent dentition reflects the difficulty in following premature children until the change of dentition.

Comparisons of the results of the present study, characterized as a cross-sectional study of specific samples, with other studies available in the literature and discussed below, must be done with caution owing to the differences in sample delineation, environmental influences, and different methodologies.

This study evaluated information from medical records of the pregnancy through behavioral and social factors of the children and their families which could impact on their oral health. The maternal disorders registered as principal causal factors for prematurity were: hypertension (42\%), preeclampsia $(14.5 \%)$, premature labor $(12.5 \%)$, anemia ( $8 \%)$, detached placenta (5\%), gestational diabetes ( $5 \%)$, and cardiopathy ( $4 \%)$. In addition to these complications, 5 births were twins and 1 birth was a pregnancy with triplets ${ }^{8}$.

In premature children, the most frequent neonatal complications were respiratory distress, hyaline membrane disease, jaundice, pneumonia, osteopenia of prematurity, anemia, and non-specific infections. Various drug therapies were necessary, among them antibiotic therapy, ventilatory assistance, parenteral nutrition, and prescription vitamin supplements containing iron and calcium. The need for these procedures was directly related to the seriousness of the general state of health of the child.

The systemic diseases in the first 3 years of the children's life, which were related by the responsible parties, were pneumonia, tonsillitis, ear infections, chicken pox, rubella, and measles. This period coincides with the time of mineralization of permanent teeth. In this study, there was no significant association between infectious diseases $(\mathrm{OR}=1.45, \mathrm{IC}=0.50-4.22)$ and rashes $(\mathrm{OR}=0.96$, $\mathrm{IC}=0.34-2.79)$ with the presence of structural defects in the enamel. However, we must not disregard the possibility of biased information. These results disagree with Chaves, et al. ${ }^{2}$ (2007), who found association between post-natal infectious episodes and the prevalence of defects in the enamel $(\mathrm{OR}=2.89, \mathrm{IC}=0.84-10.03)$. The children in group 1 (premature) and group 2 (full-term) presented mean scores from 5 to 10 permanent teeth, respectively, which should take into account the difference in mean age between the groups. These findings support the study by Seow ${ }^{18}$ (1996), in which differences between dental age and chronological age were found when children from 6 to 8 years of age, born with low birth weight, were compared with children of normal birth weight $(p<0.001)$. However, when children at 9 years of age were analyzed there was no more difference between the groups.

In the present study, approximately $72.5 \%$ of the sample presented at least one tooth with enamel defect. These findings agree with the study by Chaves, et al.2 (2007), who found a prevalence of defects in the enamel in $78.9 \%$ of the population studied. Oliveira, et al. ${ }^{14}$ (2006) found a prevalence of defects in the enamel in approximately $50 \%$ of the sample.

Lunardelli and Peres ${ }^{12}$ (2005) obtained a result of $24.4 \%$ of children with opacity and hypoplasia. However, there may have been an underestimation in the diagnoses of these patients, due to the absence of prior prophylaxis and drying of the examined surfaces.

No significant difference was found in the prevalence of enamel defects when children from G1 and G2 were divided by gender. These results coincide with those cited by Chaves, et al. ${ }^{2}$ (2007) and Oliveira, et al. ${ }^{14}$ (2006) who also found no difference.

In relation to the number of children from groups G1 and G2 who presented structural enamel defects in both dentitions, the difference was significant only for hypoplasia (Table 1 ). These results are supported by those of previous works ${ }^{1,4,5,8,11}$, who also found significant results for hypoplasia alone. From these findings, prematurity associated with low birth weight can be considered a risk factor for disorders in enamel mineralization.

In this study, the distribution of enamel defects according to dental groups revealed that they appeared most frequently in molars (9.8\%), followed by canines (9.7\%) and incisors $(8.7 \%)$. These results coincide with the study by Lunardelli and Peres ${ }^{12}$ (2005). For primary dentition, the groups of teeth most affected by hypoplasia can be presented in the following descending order: incisors $(8.0 \%)$, canines $(3.6 \%)$ and molars $(2.3 \%)$. These findings coincide with the first study, done in 2004, with the same sample ${ }^{8}$. In the present study, all primary incisors that presented hypoplasia belonged to the premature children (Table 2 ). The trauma from intubation and the calcium deficiency associated with the period of mineralization of these teeth, may have triggered the development 
of these defects $s^{4,8,18}$.

When opacity was the defect analyzed in the primary teeth, an inversion of prevalence in relation to hypoplasia occurred: molars $(7.5 \%)$, canines $(6.1 \%)$ and incisors $(0.7 \%)$ (Table 2 ). In the first study, the teeth most affected went in the following order: canines, molars and incisors. The inversion in the percentage of affected canines and molars may have been due to the greater number of teeth present in the current examination, because all the children who were examined had the 8 primary molars exposed. This is in contrast to the previous study 8 in which they presented an average of 2.7 molars. The greater prevalence of this defect in the molars and canines may be explained by the mineralization chronology of these teeth, which occurs approximately around the $9^{\text {th }}$ month of pregnancy. In children born prematurely who presented teeth with defects, the process of amelogenesis may have been interrupted or temporarily impaired. As a consequence, an enamel defect which was dependent upon the stage of amelogenesis occurring in the tooth at that moment would appear.

After categorizing the variables into the three types of defects, marked opacity appeared to be the most prevalent defect, occurring in $43 \%$ of the total sample. This was followed by hypoplasia, which occurred in $22.5 \%$ of the sample. The children in G1 presented 43 primary teeth with marked opacity (7.0\%) and 42 with hypoplasia (6.0\%). Of those in $\mathrm{G} 2,19(3.7 \%)$ and $4(0.8 \%)$ presented those defects, respectively (Figure 1). These findings disagree with those of Chaves, et al. ${ }^{2}$ (2007) and Oliveira, et al. ${ }^{14}$ (2006), who found diffuse opacity to be the most frequent defect, followed by marked opacity and hypoplasia. Lunardelli and Peres $^{12}$ (2005), analyzing a sample of 431 public preschool children, found a prevalence of $17.9 \%$ of diffuse opacity, $11.1 \%$ of hypoplasia and $6.1 \%$ of marked opacity. Many factors must be considered together in order to justify the occurrence of these defects, without discarding the possibility of diffuse opacity being due to inadequate intake of fluoride thus causing fluorosis in the examined teeth. In relation to the permanent teeth, the dental groups most affected by enamel defects went in the following order: molars (25.5\%), incisors $(21.4 \%)$ and canines $(13.6 \%)$. These findings support the study by Seow ${ }^{18}$ (1996), in which the most affected teeth were the molars followed by the incisors. As the mineralization of the permanent teeth begins at birth, or some months after the premature delivery, it may be hypothesized that persistent systemic problems lead to malformations in the enamel following birth.

Analyzing the permanent teeth affected by structural defects between the two groups, the premature children (G1) presented 43 teeth with opacity (20\%) and 2 teeth with hypoplasia (0.9\%), while in G2 there were 86 teeth with opacity $(22.7 \%)$ and 2 teeth with hypoplasia $(0.5 \%)$. The number of permanent teeth with opacity and hypoplasia between the two groups was not statistically different (Figure 2). These results disagreed with those of Seow ${ }^{18}$ (1996), who found $17 \%$ opacity and $3 \%$ hypoplasia in the group of very low birth weight, and $8 \%$ opacity and $3 \%$ hypoplasia in the group of normal birth weight, there being a statistically significant difference in the total prevalence of defects between the groups $(p<0.02)$.

The most affected permanent teeth, in a descending order, by the opacity defect in $\mathrm{G} 1$ were the molars (32.5\%) and incisors (13\%); and in G2 were the incisors (24\%), molars (20.4\%) and canines $(18.7 \%)$ (Table 3 ).

Only 3 permanent incisors presented hypoplasia in the total sample, and all had history of trauma in the homologous primary teeth.

It was observed, through multivariate analysis, that the type of birth was a risk factor for the occurrence of hypoplasia $(p=0.0034)$ in primary dentition. Premature children had 7.4 times more chance of having hypoplasia than full-term children, with a confidence interval of $95 \%$ ranging from 1.93 to 28.25 .

It is very difficult to distinguish the etiological factors for enamel alterations, because pre-, neo-, and post-natal complications may occur together, interacting among themselves. Enamel defects result from severe cumulative events associated with external factors such as social, nutritional state, and life-style questions, which must be considered because they make a strong impact on health and have repercussions on the quality of life of the individual ${ }^{3}$.

\section{CONCLUSIONS}

According to the methodology and conditions of this study, it can be concluded that there was a high prevalence of enamel defects in the total sample. The children born prematurely presented greater prevalence of hypoplasia in the primary dentition, when compared with the children born full-term. In the permanent dentition, there was no significant difference between the groups. The variables of income, level of formal education, trauma and infectious diseases did not correlate with the enamel defects in neither of the groups. Low birth weight can be considered a risk factor for defects of marked opacity and hypoplasia in the enamel only in primary dentition. 


\section{REFERENCES}

1- Aine L, Backström MC, Mäki R, Kuusela AL, Koivisto AM, Ikonen RS, et al. Enamel defects in primary and permanent teeth of children born prematurely. J Oral Pathol Med. 2000;29(8):403-9. 2- Chaves AMB, Rosenblatt A, Oliveira OF. Enamel defects and its relation to life course events in primary dentition of Brazilian children: a longitudinal study. Community Dent Health. 2007;24(1):31-6

3- Cruvinel VR, Gravina, DB, Azevedo TD, Bezerra AC, Toledo $A O$. Prevalence of dental caries and caries-related risk factors in premature and term children. Braz Oral Res. 2010;24(3):329-35. 4- Drummond BK, Ryan S, O'Sullivan EA, Congdon P, Curzon ME. Enamel defects of the primary dentition and osteopenia of prematurity. Pediatr Dent. 1992;14(2):119-21.

5- Fearne JM, Bryan EM, Elliman AM, Brook AH, Williams DM. Enamel defects in the primary dentition of children born weighing less than $2000 \mathrm{~g}$. Br Dent J. 1990;168(11):433-7.

6- Federation Dentaire Internationale. Commission on Oral Health, Research and Epidemiology. A review of the developmental defects of enamel index (DDE index). Int Dent J. 1992;42(6):411-26.

7- Franco KMD, Peres SR, Moura-Ribeiro MVL. Prenatal and neonatal variables associated with enamel hypoplasia in deciduous teeth in low birth weight preterm infants. J Appl Oral Sci. 2007;15(6):518-23.

8- Gravina DB, Cruvinel VR, Azevedo TD, Toledo OA, Bezerra AC. Prevalence of dental caries in children born prematurely or at full term. Braz Oral Res. 2006;20(4):353-7.

9- Horowitz HS. Research issues in early childhood caries. Community Dent Oral Epidemiol. 1998;26(Suppl 1):67-81.

10- Lai PY, Seow WK, Tudehope DI, Rogers Y. Enamel hypoplasia and dental caries in very-low birthweight children: a casecontrolled, longitudinal study. Pediatr Dent. 1997;19(1):42-9.

11- Li Y, Navia JM, Bian JY. Prevalence and distribution of developmental enamel defects in primary dentition of Chinese children 3-5 years old. Community Dent Oral Epidemiol. $1995 ; 23(2): 72-9$.
12- Lunardelli SE, Peres MA. Prevalence and distribution of developmental enamel defects in the primary dentition of preschool children. Braz Oral Res. 2005;19(2):144-9.

13- Norén JG, Ranggård L, Klingberg G, Persson C, Nilsson K. Intubation and mineralization in the enamel of primary teeth. Acta Odontol Scand. 1993;51(5):271-5.

14- Oliveira AF, Chaves AM, Rosenblatt A. The influence of enamel defects on the development of early childhood caries in a population with low socioeconomic status: a longitudinal study. Caries Res. 2006;40(4):296-302.

15- Sawyer DR, Nwoku AL. Malnutrition and the oral health of children in Ogbomosho, Nigeria. ASDC J Dent Child. 1985;52(2):141-5.

16- Schröder U, Lindström LG, Olsson L. Interview or questionnaire? A comparison based on the relationship between caries and dietary habits in preschoolchildren. Community Dent Oral Epidemiol. 1981;9(2):79-82.

17- Seow WK. A study of the development of the permanent dentition in very low birthweight children. Pediatr Dent. $1996 ; 18(5): 379-84$.

18- Seow WK, Humphrys C, Tudehope DI. Increased prevalence of developmental dental defects in low-birth-weight, prematurely born children: a controlled study. Pediatr Dent. 1987;9(3):221-5. 19- Silva-Sousa YTC, Peres LC, Foss MC. Are there structural alterations in the enamel organ of offspring of rats with alloxaninduced diabetes mellitus? Braz Dent J. 2003;14(3):162-7.

20- Slayton RL, Warren JJ, Kanellis MJ, Levy SM, Islam M. Prevalence of enamel hypoplasia and isolated opacities in the primary dentition. Pediatr Dent. 2001;23(1):32-6.

21- World Health Organization. International Statistical Classification of Diseases and Related Health Problems. $10^{\text {th }}$ revision. Geneva: WHO; 1999. 